Research Article

\title{
Systematically Exploring the Antitumor Mechanisms of Core Chinese Herbs on Hepatocellular Carcinoma: A Computational Study
}

\author{
Zhulin Wu, ${ }^{1}$ Li He, ${ }^{2}$ Lianan Wang, ${ }^{2}$ and Lisheng Peng $\mathbb{D}^{2}$ \\ ${ }^{1}$ The Fourth Clinical Medical College of Guangzhou University of Chinese Medicine, Shenzhen 518033, China \\ ${ }^{2}$ Shenzhen Traditional Chinese Medicine Hospital, Shenzhen 518033, China \\ Correspondence should be addressed to Lisheng Peng; szlisheng_peng@163.com
}

Received 2 May 2020; Accepted 8 September 2020; Published 15 September 2020

Academic Editor: Jamal A. Mahajna

Copyright (c) 2020 Zhulin Wu et al. This is an open access article distributed under the Creative Commons Attribution License, which permits unrestricted use, distribution, and reproduction in any medium, provided the original work is properly cited.

Objective. Chinese herbs play a positive role in the management of hepatocellular carcinoma (HCC) in China. However, it is not clear which of Chinese herbs are critical for the treatment of HCC. Besides, mechanisms of CCHs in the treatment of HCC remain unclear. Hence, our goal is to identify the core Chinese herbs (CCHs) for treating HCC and explore their antitumor mechanism. Methods. Firstly, clinical traditional Chinese medicine (TCM) prescriptions for HCC were collected from Chinese National Knowledge Infrastructure (CNKI) database, and then, data mining software was used to identify CCHs. After that, bioactive compounds and corresponding target genes of CCHs were obtained using three TCM databases, and target genes of HCC were acquired from MalaCards and OMIM. Subsequently, common target genes of CCHs and HCC were screened. Moreover, biological functions and pathways were analyzed, and Cytoscape plugin cytoHubba was used to identify hub genes. Finally, prognostic values of hub genes were verified by survival analysis, and the molecular docking approach was utilized to validate the interactions between targets and bioactive compounds of CCHs. Results. Eight CCHs were determined from 630 prescriptions, and 100 bioactive compounds (e.g., quercetin and luteolin) and 126 common target genes were screened. Furthermore, common target genes of $\mathrm{CCH}$ and $\mathrm{HCC}$ were mainly enriched in cancer-associated pathways, and six hub genes with statistical significance in survival analysis were selected as key target genes for molecular docking. Additionally, molecular docking showed that the bioactive compounds docked well with the protein receptors of key target genes. Conclusion. By combining data mining, network pharmacology, molecular docking, and survival analysis methods, we found that CCHs may play a therapeutic role in HCC through regulating the target genes and pathways related to cancer occurrence and development, angiogenesis, metastasis, and prognosis.

\section{Introduction}

As one of the most common cancers, hepatocellular carcinoma (HCC) is characterized by high morbidity and mortality rates. HCC is predicted to be the sixth most frequent cancer and the fourth most common cause of cancer death worldwide in 2018 [1]. In addition, advancedstage HCC has a 5 -year survival rate of only $5-15 \%$ [2]. The chief etiologies for HCC include chronic infection with hepatitis B virus (HBV) or hepatitis C virus (HCV), alcoholic liver disease, and nonalcoholic fatty liver disease [3]. Currently, the therapeutic options for patients with HCC are poor and mainly include surgery, liver transplantation, chemoembolization, and molecularly targeted therapy $[4,5]$. Moreover, most patients with late-stage HCC have lost the opportunity of surgical resection, and not all patients with advanced HCC are suitable for chemotherapy or targeted therapy. Thus, it is necessary to develop novel approaches for HCC control.

More and more researchers are paying significant interest in traditional Chinese medicine (TCM) which has been used to treat cancer in China for a long time [6]. Furthermore, TCM is effective in improving symptoms, reducing side effects of chemotherapy, suppressing cancer 
cell growth, and regulating key intracellular signaling pathways [7]. As an important part of TCM, Chinese herbs play a positive role in the management of HCC in China [8]. Previous literature indicated that several TCM prescriptions consisting of Chinese herbs had anti-HCC effects in both basic and clinical research $[9,10]$. Moreover, evidence demonstrated that TCM combined with chemotherapy showed significant efficacy and safety in improvement of life quality and reduction of chemotherapy side effects [11]. Nevertheless, TCM prescriptions for treating HCC are often based on the experience of TCM doctors, and it is unclear which core Chinese herbs (CCHs) are effective treatments for HCC. Additionally, the molecular mechanisms underlying the anti-HCC activity of $\mathrm{CCHs}$ are not completely clear.

Currently, data mining analysis of TCM prescriptions has become a research focus in the TCM field [12], which can be used to identify CCHs from a large number of clinical prescriptions. In the past, the complicated interactions between "multi-components" and "multi-targets" of Chinese herbs hindered the mechanism study of these herbs. At present, network pharmacology provides an effective solution to overcome these obstacles and can reveal the synergistic effects of complex Chinese herbs on human systems from a holistic view [13]. In the present study, data mining software was applied to determine $\mathrm{CCH}$ s for the treatment of HCC, and mechanisms of CCHs on HCC were analyzed by network pharmacology. Besides, survival analysis and molecular docking methods were used to validate the results of network pharmacology analysis.

\section{Materials and Methods}

2.1. Data Mining of TCM Prescriptions. Clinical TCM prescriptions intended solely for HCC were collected from studies in the Chinese National Knowledge Infrastructure (CNKI) database. The search was conducted using the following search terms: "(Chinese medicine OR prescription OR decoction) AND (hepatocellular carcinoma OR liver cancer) AND clinical" (date: 1979 to 3 March 2020). The inclusion criteria for the studies included the following: (1) the first diagnosis of cancer patients being HCC; (2) clinical research on oral TCM prescriptions or oral TCM prescriptions combined with Western medicine in the treatment of HCC; (3) experience of TCM experts. Besides, exclusion criteria were as follows: (1) repeated literature and animal experiments; (2) TCM prescriptions in the literature being primarily for treating acute symptoms (e.g., cold and cough); (3) external prescriptions or Chinese patent medicine. Subsequently, eligible studies were screened, and TCM prescriptions were extracted from the included studies. Furthermore, Traditional Chinese Medicine Inheritance Support System (TCMISS, from the Institute of Chinese Materia Medica, China Academy of Chinese Medical Sciences, version 2.5) was employed to identify the CCHs from all prescriptions. TCMISS software has been widely used to analyze TCM prescriptions, and it has the function of text mining, association rules, and complex system entropy clustering methods [12]. In the present study, association rules in TCMISS were utilized to determine CCHs under the condition of support degree $\geq 126$ (20\%).

2.2. Screening Bioactive Compounds and Target Genes. Bioactive compounds and corresponding targets of CCHs were obtained using Traditional Chinese Medicine Systems Pharmacology Database and Analysis Platform (TCMSP, http://tcmspw.com/tcmsp.php) [14], Integrative Pharmacology-Based Research Platform of TCM (TCMIP, http:// www.tcmip.cn/) [15], and Bioinformatics Analysis Tool for Molecular Mechanism of TCM (BATMAN-TCM, http:// bionet.ncpsb.org/batman-tcm/) [16]. In this study, all TCM prescriptions composed of Chinese herbs were given orally, and the bioactive compounds were selected under the conditions of drug-likeness (DL) $\geq 0.18$ (mean value for all molecules within the DrugBank database) and oral bioavailability $(\mathrm{OB}) \geq 30 \%$ [17]. Additionally, all compounds are numbered using Mol ID from TCMSP, and all target names were converted to official gene names using the UniProt database (https://www.uniprot.org/). Moreover, potential therapeutic target genes associated with $\mathrm{HCC}$ were obtained from the MalaCards (https://www.malacards.org/) [18] and OMIM databases (https://omim.org/) [19] using "hepatocellular carcinoma" as the keyword, and known target genes for HCC were screened after removing duplicates.

2.3. Constructing the Network of CCHs and Targets. Venn diagrams were used to determine common target genes of CCHs and HCC by "VennDiagram" package in R 3.6.0 (https://www.r-project.org/). Then, the interaction network of CCHs bioactive compounds and common target genes was built using Cytoscape 3.7.1 (https://cytoscape.org/), and the relationships between bioactive compounds of $\mathrm{CCHs}$ and common target genes were displayed in the interaction network.

2.4. Signaling Pathway and Gene Ontology Analyses. Gene Ontology (GO) and Kyoto Encyclopedia of Genes and Genomics (KEGG) pathway analyses for common target genes were done using the Database for Annotation, Visualization, and Integrated Discovery (DAVID, https:// david.ncifcrf.gov/) [20], and false discovery rate (FDR) $<0.05$ was accepted as significant. In addition, GO analysis was performed according to three categories, namely, biological process (BP), cellular component (CC), and molecular function (MF), and the results were shown as a bar plot using the "ggplot2" package in R 3.6.0.

2.5. Protein-Protein Interaction Analysis and Screening for $\mathrm{Hub}$ Genes. Protein-protein interaction (PPI) network analysis of the common target genes was completed using Search Tool for the Retrieval of Interacting Genes (STRING) database (https://string-db.org/) [21] with the highest confidence (score $>0.9$ ). Then, hub genes were screened from the PPI network by degree, maximum neighborhood component (MNC), and maximal clique centrality (MCC) algorithms in the Cytoscape 3.7.1 plugin, cytoHubba [22, 23]. 
The overlap genes, predicted by all three algorithms, were selected as hub genes.

2.6. Evaluation of Prognostic Values of Hub Genes. The prognostic values of hub genes were assessed by survival analysis using the Kaplan-Meier plotter (http://kmplot.com/ analysis/) [24]. The Kaplan-Meier plotter includes data on HCC survival in the Cancer Genome Atlas (TCGA). In survival analysis, overall survival (OS) was analyzed by the Kaplan-Meier (KM) method (log-rank test), and a $p$ value less than 0.05 was considered to be a significant difference. In this study, the hub genes that displayed statistically significant differences were considered to be the key target genes.

\subsection{Molecular Docking of Key Target Proteins and Bioactive} Compounds. In the present study, molecular docking was used to verify the interactions between protein receptors of key target genes and bioactive compounds of CCHs. The corresponding protein receptors of key target genes were acquired using Protein Data Bank (PDB) database (https:// www.rcsb.org/) [25], and we included protein receptors based on the following criteria: (1) The structure of protein receptors was determined by X-ray diffraction approach. (2) X-ray resolution $<3 \AA$ was preferred. (3) Protein structure with initial ligand was also preferred. AutoDockTools (version 1.5.6, http://autodock.scripps.edu) was utilized to remove the original ligands (if any), excess protein chains, and water molecules of protein receptors [26], and then hydrogens were added to the protein receptors. Furthermore, grid boxes in AutoDockTools were used to identify the docking coordinates. After that, bioactive compounds of $\mathrm{CCHs}$ corresponding to key targets were used as ligands, and the "mol2" files of compounds were obtained using TCMSP. Moreover, the files of protein receptors and ligands were converted into "PDBQT" format using AutoDockTools. Finally, AutoDock Vina program (http://vina.scripps.edu/) [27] was used to dock bioactive compounds into the corresponding protein receptors. Besides, docking results were analyzed and visualized using PyMOL (http://www.pymol. org/) and Discovery Studio 2016 (BIOVIA, San Diego, USA). The workflow of our study is displayed in Figure 1.

\section{Results}

3.1. Results of Data Mining. A total of 1472 studies were identified through CNKI database search. According to the criteria described above, $630 \mathrm{TCM}$ prescriptions for the treatment of HCC were obtained from 527 studies. In addition, the result of TCMISS analysis showed that there were 180 different Chinese herbs used in all prescriptions, and a total of 19 combinations of Chinese herbs that were most commonly used in the TCM prescriptions were found (Table 1). Then, nine CCHs were identified by TCMISS, namely, Largehead Atractylodes Rhizome (Bai Zhu), Poria (Fu Ling), Radix Bupleuri (Chai Hu), Radix Astragali (Huang Qi), Herba Hedyotis (Bai Hua She She Cao), Radix Codonopsis (Dang Shen), Radix Paeoniae Alba (Bai Shao), Radix Glycyrrhizae (Gan Cao), Herba Scutellariae Barbatae
(Ban Zhi Lian), which were shown in a network (Figure 2(a)). According to the theory of TCM, Radix Glycyrrhizae (Gan Cao) is often used as a harmonizing (Tiao $\mathrm{He}$ ) drug, so it was not included in the following analysis. Finally, excluding Radix Glycyrrhizae (Gan Cao), eight $\mathrm{CCHs}$ were selected for further analysis.

3.2. Bioactive Compounds and Targets. Bioactive compound counts of each core Chinese herb from the three databases are shown in Table 2. After merging the data and removing duplicates, a total of 100 bioactive compounds were identified, and 840 target genes of eight CCHs were identified. Additionally, 191 and 745 HCC-related target genes were obtained from OMIM and MalaCards databases, respectively. Following the removal of duplicates, a total of 918 therapeutic target genes for HCC were found.

3.3. Network of Bioactive Compounds and Common Targets. A total of 126 common target genes of CCHs and HCC were identified using Venn diagram (Figure 2(b)), and the interaction network of $\mathrm{CCHs}$ bioactive compounds and common target genes was established, including 198 nodes (72 bioactive compounds and 126 genes) and 510 edges (Figure 3). As shown in Figure 3, eight CCHs were divided into 3 types: health-strengthening (Fu Zheng), heat-clearing and detoxicating (Qingre Jiedu), and relieving liver Qi stagnation (Shu Gan). Furthermore, the top 30 bioactive compounds are presented in Table 3 according to the gene count, and the results showed that quercetin, stigmasta-5,22dien-3-one, luteolin, wogonin, kaempferol, beta-sitosterol, baicalein, stigmasterol, pyrethrin II, etc. connected with most of the common target genes.

3.4. GO and KEGG Analyses Results. The result of GO analysis revealed that common target genes were mainly enriched in negative regulation of apoptotic process, positive regulation of gene expression, response to drug, positive regulation of transcription from RNA polymerase II promoter, and cellular response to hypoxia (BP); enzyme binding, protein binding, transcription factor binding, identical protein binding, and protein heterodimerization activity (MF); cytosol, nucleus, cytoplasm, nucleoplasm, and phosphatidylinositol 3-kinase complex (CC). Top 5 GO terms of each category are shown in Figure 4. Besides, KEGG pathway analysis demonstrated that most common target genes were related to pathways in cancer, proteoglycans in cancer, hepatitis B, PI3K-Akt, HIF-1 signaling pathway, etc., and the results of the top 20 signaling pathways are listed in Table 4.

3.5. PPI Analysis and Hub Genes. The PPI network with 126 nodes and 698 edges was constructed by STRING and visualized by Cytoscape v. 3.7.1 (Figure 5(a)). Then, hub genes were identified based on MNC, degree, and MCC methods (Figure 5(b)). The interaction network of eight hub genes is shown in Figure 5(c). According to the result of cytoHubba 


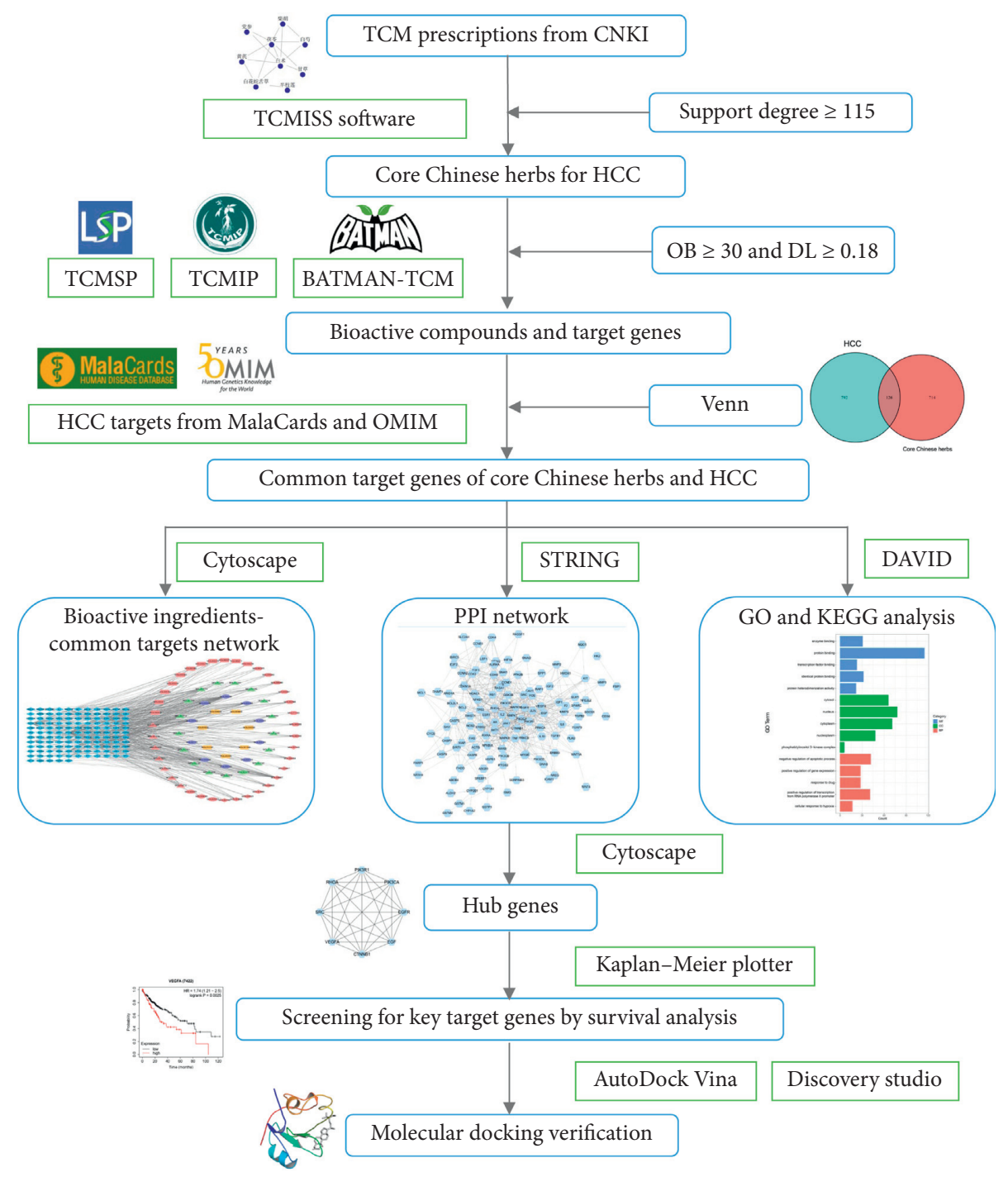

FIgURE 1: Flowchart of the present study.

in Cytoscape, the eight hub genes included SRC, PIK3CA, RHOA, PIK3R1, EGFR, VEGFA, EGF, and CTNNB1.

3.6. Results of Survival Analysis. KM survival curves showed that high expressions of PIK3R1 and EGFR were correlated with longer $\mathrm{OS}$ in patients with HCC, and high expressions of SRC, RHOA, VEGFA, and EGF were associated with shorter OS. Additionally, CTNNB1 and PIK3CA showed no significant differences. The results of survival analysis for the hub genes are presented in Figures 6(a)-6(h). Moreover, six hub genes with statistical significance are defined as key target genes and selected for molecular docking.

3.7. Results of Molecular Docking. After validating the prognostic values of key genes, protein receptors of key target genes were chosen for molecular docking with corresponding six bioactive compounds of CCHs. The affinity binding values of molecular dockings calculated by AutoDock Vina are demonstrated in Table 5. In the present study, the binding between the receptor (protein receptor of key target gene) and the ligand (bioactive compound) was considered to be good if the affinity value $<-5.0 \mathrm{kcal} / \mathrm{mol}$, and the lower the affinity value was, the higher the affinity of the bioactive compound and the protein receptor was. As shown in Table 5, stigmasta-5,22-dien-3-one, luteolin, quercetin, pyrethrin II, palbinone, and baicalein had strong binding affinities for the corresponding proteins, and molecular docking results are shown in Figures 7(a)-7(f).

\section{Discussion}

According to the data mining results, eight Chinese herbs, including Largehead Atractylodes Rhizome, Poria, Radix Bupleuri, Radix Astragali, Herba Hedyotis, Radix Codonopsis, Radix Paeoniae Alba, and Herba Scutellariae Barbatae, were identified as the CCHs for the treatment of HCC. Based on the TCM theory, it is believed that the pathogenesis of HCC is due to "deficiency of healthy Qi," "liver Qi stagnation," "heat-toxicity," etc. In TCM, Largehead 
TABle 1: Top 19 commonly used combinations of Chinese herbs according to the association rules.

\begin{tabular}{lcc}
\hline No. & The combinations of Chinese herbs & Freq. \\
\hline 1 & Largehead Atractylodes Rhizome, Poria & 284 \\
2 & Radix Astragali, Largehead Atractylodes Rhizome & 195 \\
3 & Radix Codonopsis, Largehead Atractylodes Rhizome & 188 \\
4 & Radix Codonopsis, Poria & 170 \\
5 & Largehead Atractylodes Rhizome, Radix Bupleuri & 163 \\
6 & Radix Bupleuri, Poria \\
7 & Radix Astragali, Poria \\
8 & Radix Codonopsis, Largehead Atractylodes Rhizome, Poria \\
9 & Largehead Atractylodes Rhizome, Radix Paeoniae Alba \\
10 & Radix Paeoniae Alba, Radix Bupleuri \\
11 & Herba Hedyotis, Largehead Atractylodes Rhizome \\
12 & Largehead Atractylodes Rhizome, Radix Glycyrrhizae \\
13 & Herba Hedyotis, Poria \\
14 & Radix Astragali, Largehead Atractylodes Rhizome, Poria \\
15 & Radix Glycyrrhizae, Poria \\
16 & Largehead Atractylodes Rhizome, Radix Bupleuri, Poria \\
17 & Herba Hedyotis, Radix Astragali \\
18 & Radix Paeoniae Alba, Poria \\
19 & Herba Hedyotis, Herba Scutellariae Barbatae & 160 \\
\hline
\end{tabular}

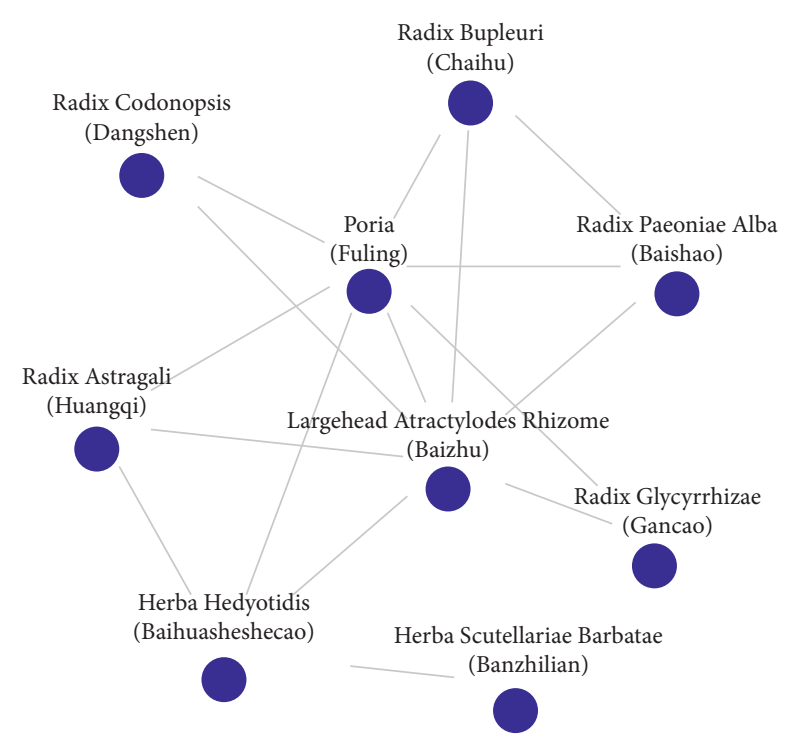

(a)

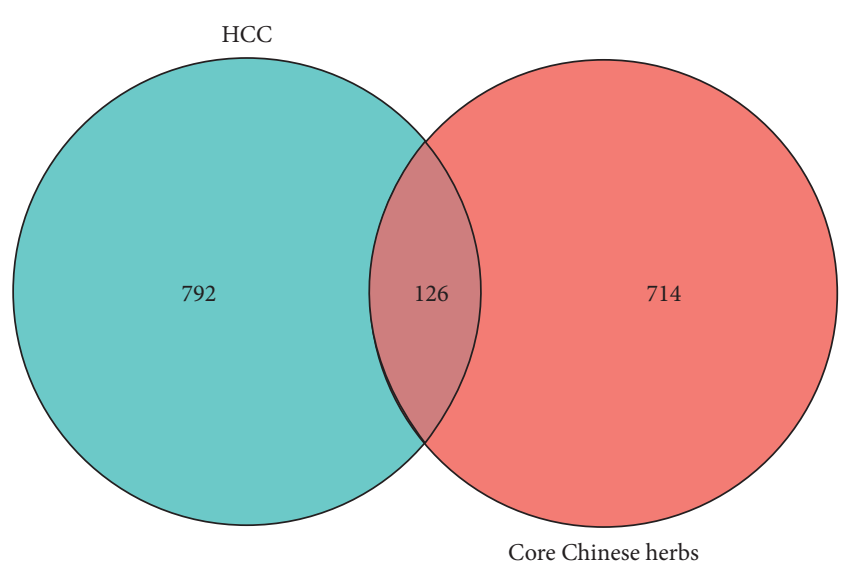

Core Chinese herbs

(b)

Figure 2: The network of CCHs and the Venn diagram of HCC and CCHs. (a) There are 16 edges and nine nodes in the network. Each edge represents a direct combination of $\mathrm{CCHs}$, and each node represents a core Chinese herb. (b) Venn diagrams showing overlapping target genes between HCC and CCHs.

TABLE 2: Information on bioactive compounds from three TCM databases.

\begin{tabular}{|c|c|c|c|c|}
\hline $\mathrm{CCHs}$ & TCMSP & TCMIP & BATMAN-TCM & Total \\
\hline Largehead Atractylodes Rhizome & 7 & 3 & 1 & 7 \\
\hline Poria & 15 & 7 & 6 & 18 \\
\hline Radix Bupleuri & 17 & 2 & 10 & 18 \\
\hline Radix Astragali & 20 & 5 & 9 & 25 \\
\hline Herba Hedyotis & 7 & - & 1 & 7 \\
\hline Radix Codonopsis & 21 & 4 & 12 & 24 \\
\hline Radix Paeoniae Alba & 13 & 7 & 9 & 18 \\
\hline Herba Scutellariae Barbatae & 29 & 5 & 5 & 32 \\
\hline
\end{tabular}

CCHs: core Chinese herbs; TCMSP: Traditional Chinese Medicine Systems Pharmacology Database and Analysis Platform; TCMIP: Integrative Pharmacology-Based Research Platform of TCM; BATMAN-TCM: Bioinformatics Analysis Tool for Molecular Mechanism of TCM. 


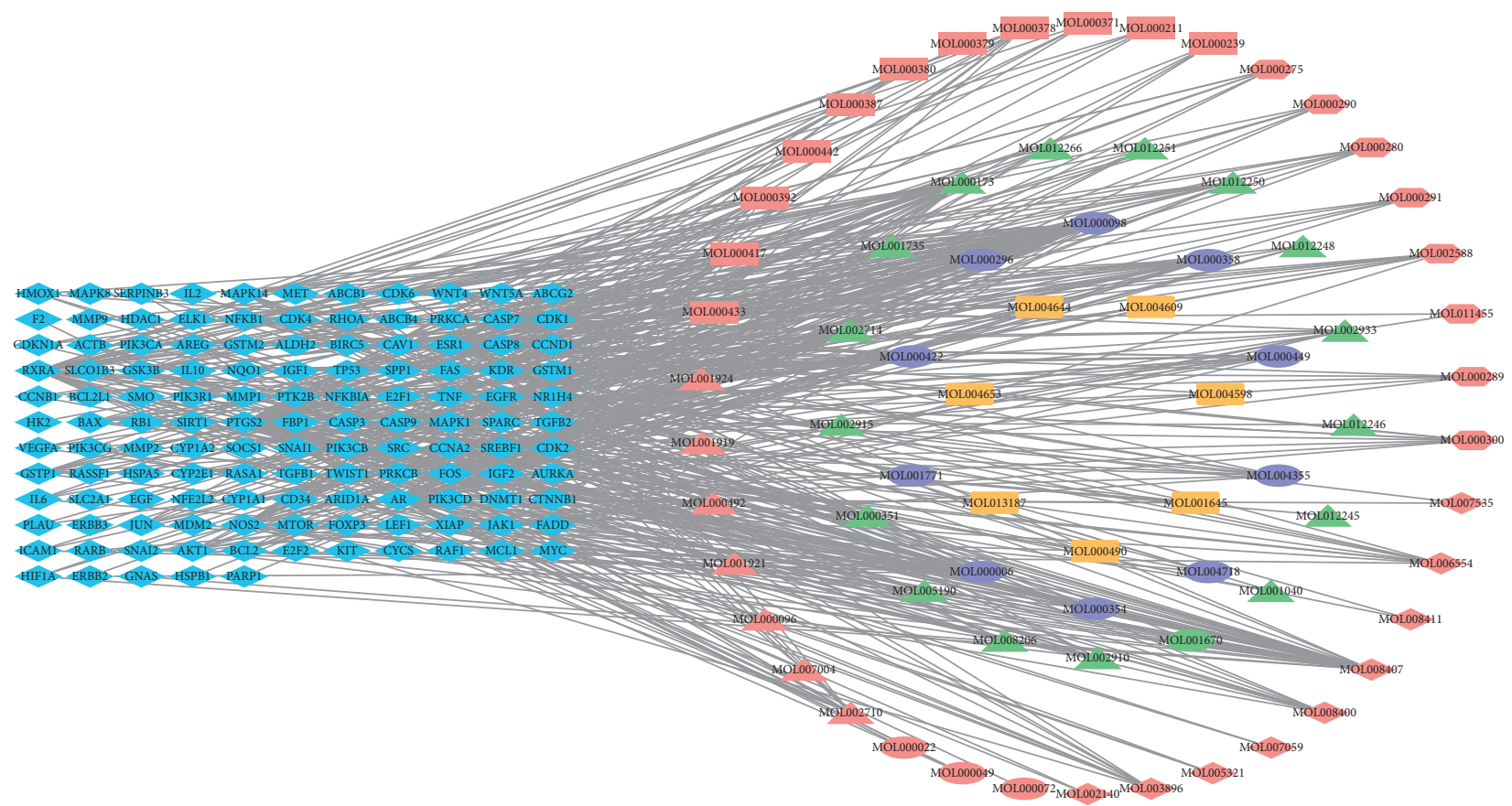

FIGURE 3: A network of common target genes and CCHs bioactive compounds. In the network, blue diamonds represent the common target genes. Pink, green, and yellow represent the compounds of health-strengthening (Fu Zheng) CCHs, heat-clearing and detoxicating (Qingre Jiedu) CCHs, and relieving liver Qi stagnation (Shu Gan) CCHs, respectively. In the pink shapes, triangles, ellipses, diamonds, hexagons, and rectangles stand for the compounds of Radix Paeoniae Alba, Largehead Atractylodes Rhizome, Radix Codonopsis, Poria, and Radix Astragali, respectively. In green shapes, ellipses and triangles represent the compounds of Herba Hedyotis and Herba Scutellariae Barbatae, respectively. Yellow rectangles represent the compounds of Radix Bupleuri. Purple ellipses indicate common compounds of multiple CCHs.

Atractylodes Rhizome, Poria, Radix Astragal, Radix Codonopsis, and Radix Paeoniae Alba can be used for "supporting the healthy energy"; Herba Hedyotis and Herba Scutellariae Barbatae have the effect of "clearing heat and removing toxicity"; Radix Bupleuri can be used for "relieving liver Qi stagnation." Thus, data mining results were highly consistent with the TCM theory. In addition, the bioactive compounds-common targets network showed that the critical bioactive compounds of CCHs could be quercetin, stigmasta-5,22-dien-3-one, luteolin, wogonin, kaempferol, betasitosterol, baicalein, Stigmasterol, Pyrethrin II, formononetin, (+)-catechin, and palbinone according to the gene counts (genes $\geq 10$ ). Previous studies indicated that quercetin and luteolin could inhibit the growth of HCC cells and enhance chemotherapy efficacy $[28,29]$. Also, wogonin and kaempferol could effectively suppress the proliferation and invasion of HCC cells by regulating EGFR signaling pathways and $\mathrm{PI} 3 \mathrm{~K} / \mathrm{AKT} / \mathrm{mTOR}$ pathway, respectively $[30,31]$. Stigmasterol, beta-sitosterol, and baicalein have been found to induce apoptosis of HCC cells by upregulating proapoptotic gene (Bax) and downregulating antiapoptotic gene (Bcl-2) [32-34]. Experimental research showed that formononetin could impede the epithelial-mesenchymal transition (EMT) and malignant progression of HCC [35]. Besides, (+)-catechin could inhibit the proliferation of HCC cells via the caspase-dependent pathway [36]. Overall, previous studies strongly support our findings.

The results of GO analysis showed that common target genes were significantly involved in cellular components and biological processes which are related to cell apoptosis, proliferation, differentiation, and various cellular functions. Moreover, the molecular functions of common target genes may be correlated with the physiological and metabolic processes of liver. KEGG analysis revealed that most of the common target genes were mainly enriched in cancerrelated signaling pathways. Previous literature reported that the PI3K/Akt pathway was activated in $30-50 \%$ of HCC and the upregulation of p-Akt was correlated with poor survival, metastasis, and vascular invasion in HCC patients $[37,38]$. Therefore, PI3K/Akt pathway could shed light on a novel strategy for drug development for HCC. Furthermore, a positive correlation between HBV infection and HCC was observed, and HBV infection can result in the activation of protooncogenes and inactivation of tumor suppressor genes [39, 40]. Proteoglycans are extracellular matrix components of liver microenvironment which play an important role in the progression of HCC and have the potential to be the HCC therapeutic target [41]. It is also reported that kindlin-2 is a member of the focal adhesion protein family which promotes HCC invasion, metastasis [42]. MicroRNA dysregulation has been found to be involved in all stages of HCC, and some microRNAs, such as miR-17-92, miR-21, and miR-221, are generally upregulated in HCC [43]. In addition, HIF-1 plays a critical role in immune escape and EMT of HCC [44]. Literature has shown that the interaction between thyroid hormone and its receptor plays an important role in the regulation of development and proliferation, and metastasis of HCC 
TABLE 3: Data of the top 30 bioactive compounds in CCHs (gene count $\geq 6$ ).

\begin{tabular}{|c|c|c|c|c|c|}
\hline Mol ID & Bioactive compounds & $\mathrm{OB}$ & $\mathrm{DL}$ & Genes & Database \\
\hline MOL000098 & Quercetin & 46.43 & 0.28 & 58 & TCMSP/BATMAN \\
\hline MOL008407 & Stigmasta-5,22-dien-3-one & 45.40 & 0.76 & 33 & TCMSP/TCMIP/BATMAN \\
\hline MOL000006 & Luteolin & 36.16 & 0.25 & 31 & TCMSP \\
\hline MOL000173 & Wogonin & 30.68 & 0.23 & 26 & TCMSP/TCMIP \\
\hline MOL000422 & Kaempferol & 41.88 & 0.24 & 19 & TCMSP/BATMAN \\
\hline MOL000358 & Beta-sitosterol & 36.91 & 0.75 & 16 & TCMSP/BATMAN \\
\hline MOL002714 & Baicalein & 33.52 & 0.21 & 14 & TCMSP \\
\hline MOL000449 & Stigmasterol & 43.83 & 0.76 & 12 & TCMSP/TCMIP/BATMAN \\
\hline MOL002710 & Pyrethrin II & 48.36 & 0.35 & 12 & TCMIP/BATMAN \\
\hline MOL000392 & Formononetin & 69.67 & 0.21 & 10 & TCMSP/BATMAN \\
\hline MOL000492 & $(+)$-Catechin & 54.83 & 0.24 & 10 & TCMSP/TCMIP/BATMAN \\
\hline MOL001919 & Palbinone & 43.56 & 0.53 & 10 & TCMSP/TCMIP/BATMAN \\
\hline MOL000378 & 7-O-Methylisomucronulatol & 74.69 & 0.30 & 9 & TCMSP \\
\hline MOL000417 & Calycosin & 47.75 & 0.24 & 9 & TCMSP/BATMAN \\
\hline MOL003896 & 7-Methoxy-2-methyl isoflavone & 42.56 & 0.20 & 9 & TCMSP \\
\hline MOL004355 & Spinasterol & 42.98 & 0.76 & 9 & TCMSP/BATMAN \\
\hline MOL006554 & Taraxerol & 38.40 & 0.77 & 9 & TCMSP/TCMIP/BATMAN \\
\hline MOL008400 & Glycitein & 50.48 & 0.24 & 9 & TCMSP/BATMAN \\
\hline MOL012250 & 7-Hydroxy-5,8-dimethoxyflavone & 43.72 & 0.25 & 9 & TCMSP/TCMIP/BATMAN \\
\hline MOL000300 & Dehydroeburicoic acid & 44.17 & 0.83 & 8 & TCMIP/BATMAN \\
\hline MOL000351 & Rhamnazin & 47.14 & 0.34 & 8 & TCMSP \\
\hline MOL000354 & Isorhamnetin & 49.60 & 0.31 & 8 & TCMSP/BATMAN \\
\hline MOL002588 & Eburicol & 42.37 & 0.77 & 8 & BATMAN \\
\hline MOL002910 & Carthamidin & 41.15 & 0.24 & 8 & TCMIP/BATMAN \\
\hline MOL002933 & 5,7,4'-Trihydroxy-8-methoxyflavone & 36.56 & 0.27 & 8 & TCMIP/BATMAN \\
\hline MOL004644 & Sainfuran & 79.91 & 0.23 & 8 & BATMAN \\
\hline MOL008206 & Moslosooflavone & 44.09 & 0.25 & 7 & TCMSP \\
\hline MOL000275 & Trametenolic acid & 38.71 & 0.80 & 6 & TCMSP/TCMIP \\
\hline MOL000280 & Dehydrotumulosic acid & 31.07 & 0.82 & 6 & TCMIP \\
\hline MOL012266 & Rivularin & 37.94 & 0.37 & 6 & TCMSP/TCMIP \\
\hline
\end{tabular}

DL: drug-likeness; OB: oral bioavailability; TCMSP: Traditional Chinese Medicine Systems Pharmacology Database and Analysis Platform; TCMIP: Integrative Pharmacology-Based Research Platform of TCM; BATMAN: Bioinformatics Analysis Tool for Molecular Mechanism of TCM.

[45]. Research also showed that the occurrence of HBVrelated HCC activates the Ras/MAPK signaling pathway which is correlated with a poor prognosis $[46,47]$. Besides, patients with cancer are more likely to suffer from various infections due to low immune function, which may be related to HTLV-I infection, Chagas disease, and Influenza A pathways. Taken together, our results are in line with previous studies, suggesting that $\mathrm{CCH}$ s may exert their antitumor effect in HCC by regulating the occurrence, progression, angiogenesis, and metastasis of HCC.

Based on the results of the PPI network and survival curves, we identify six key target genes of CCHs in the treatment of HCC, namely, SRC, RHOA, PIK3R1, EGFR, VEGFA, and EGF. An experiment showed that HBV core protein could promote tumorigenesis of HCC cells by upregulating the expression of SRC protooncogene and then activating SRC/PI3K/Akt pathway [48]. A previous study demonstrated that RHOA (Ras homolog gene family member A) is commonly overexpressed in HCC, and its expression is associated with poor prognosis [49]. Additionally, evidence showed that knockdown of PIK3R1 promoted apoptosis of HCC cells and downregulated p-PI3K and p-AKT expressions in HCC cells [50]. As a growth factor, EGF plays a crucial role in cell proliferation and migration by binding to its receptor EGFR, and high expression of EGF could induce highly malignant HCC [51]. Previous studies suggested that EGFR is overexpressed or mutated in HCC and may be closely related to the formation, invasive growth, and clinical characteristics of HCC $[52,53]$. Angiogenesis is closely related to tumor growth and invasion, and HCC is recognized as a typical angiogenic tumor [54]. VEGFA is an inducer of angiogenesis in HCC, and the expression of VEGFA in HCC was significantly higher than that in normal liver tissues [55]. Overall, previous studies made our results more reliable to some extent.

The results of network pharmacology were also confirmed by molecular docking. Table 5 shows that the affinity values of all docking results were less than $-5.0 \mathrm{kcal} / \mathrm{mol}$, which indicated that the protein receptors of nine key target genes were docked well with the six different compounds of CCHs. As shown in the three-dimensional mode of Figure 7, six active compounds were successfully docked to the active pocket of protein receptors (SRC, PIK3R1, EGFR, RHOA, VEGFA, EGF), and the two-dimensional diagram in Figure 7 also demonstrates the interactions between active compounds and protein receptors. According to our findings, van der Waals forces, hydrogen bonds, Alkyl, $\pi$-Alkyl, $\pi$-Cation, $\pi$-Sigma, etc. were shown to be involved in the interactions between receptors and compounds. For 


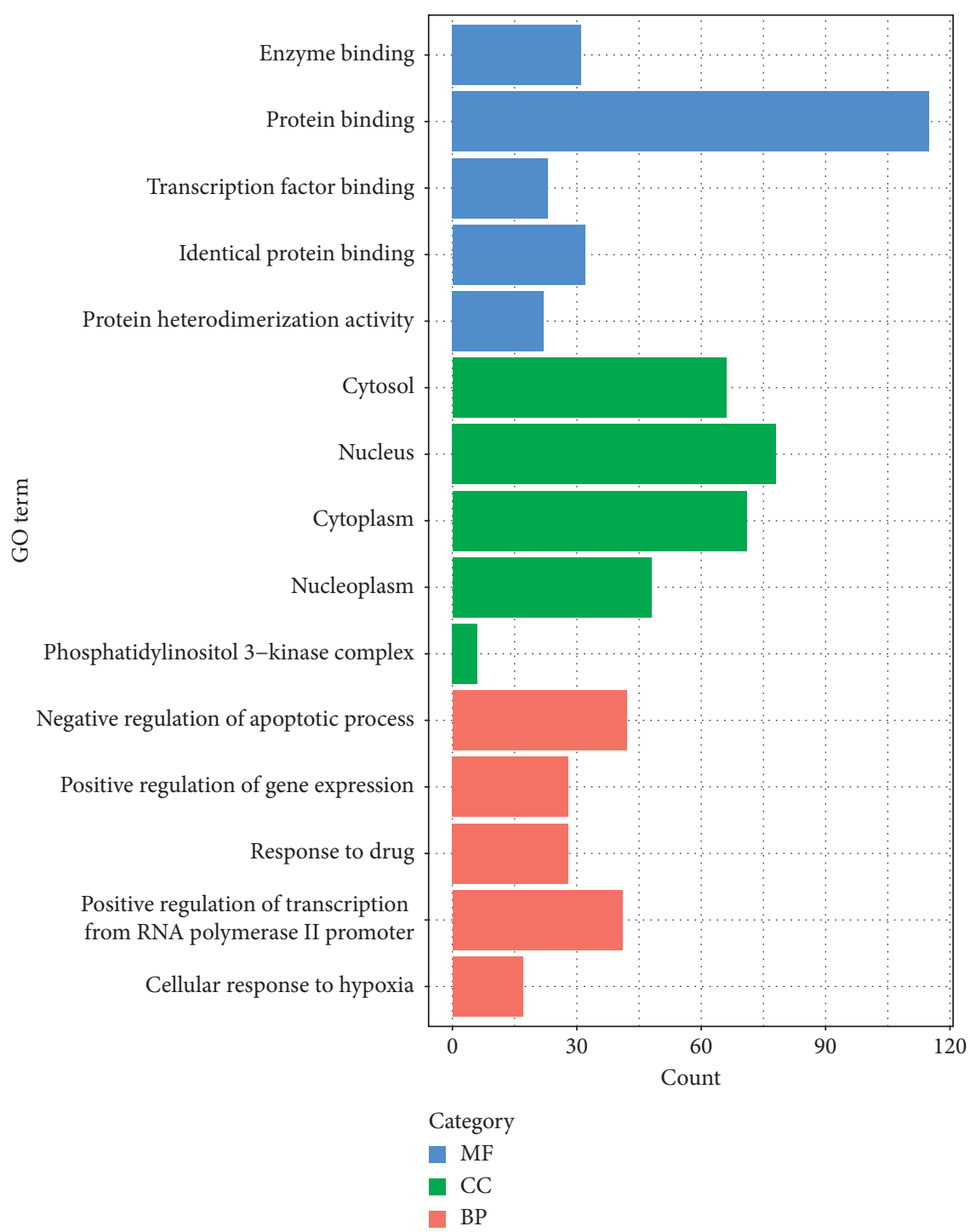

Figure 4: Top five significantly enriched GO terms of each category in GO analysis.

TABLE 4: Results of KEGG pathways (top 20).

\begin{tabular}{lcrr}
\hline ID & KEGG pathways & Gene count & FDR \\
\hline hsa05200 & Pathways in cancer & 70 & $1.13 E-51$ \\
hsa05205 & Proteoglycans in cancer & 45 & 44 \\
hsa05161 & Hepatitis B & 36 & $9.76 E-40$ \\
hsa04151 & PI3K-Akt signaling pathway & 34 & $7.78 E-15$ \\
hsa05166 & HTLV-I infection & 32 & $3.92 E-17$ \\
hsa05206 & MicroRNAs in cancer & 30 & $1.44 E-13$ \\
hsa04510 & Focal adhesion & 29 & $1.17 E-15$ \\
hsa05215 & Prostate cancer & 26 & $1.75 E-25$ \\
hsa05210 & Colorectal cancer & 26 & $1.69 E-25$ \\
hsa05212 & Pancreatic cancer & 26 & $7.14 E-25$ \\
hsa05222 & Small cell lung cancer & 26 & $1.79 E-21$ \\
hsa04919 & Viral carcinogenesis & 26 & $6.72 E-18$ \\
hsa05203 & Nonroid hormone signaling pathway & 24 & $1.37 E-11$ \\
hsa05223 & Chronic myeloid leukemia & 24 & $1.81 E-23$ \\
hsa05220 & HIF-1 signaling pathway & 24 & $1.75 E-20$ \\
hsa04066 & Chagas disease & 24 & $2.71 E-17$ \\
hsa05142 & Influenza A & 24 & $1.92 E-16$ \\
hsa05164 & Ras signaling pathway & 24 & $3.13 E-11$ \\
hsa04014 & Glioma & 23 & $8.72 E-09$ \\
hsa05214 & & $4.03 E-20$ \\
\hline
\end{tabular}




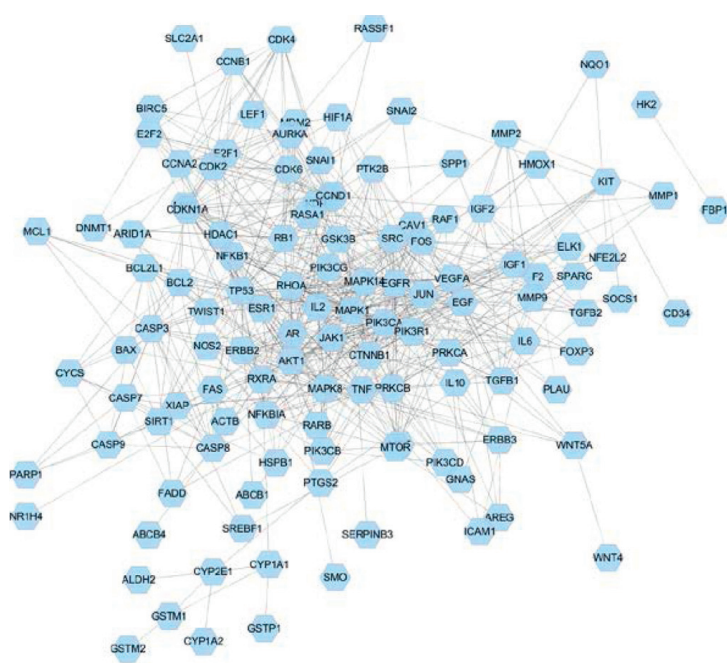

(a)

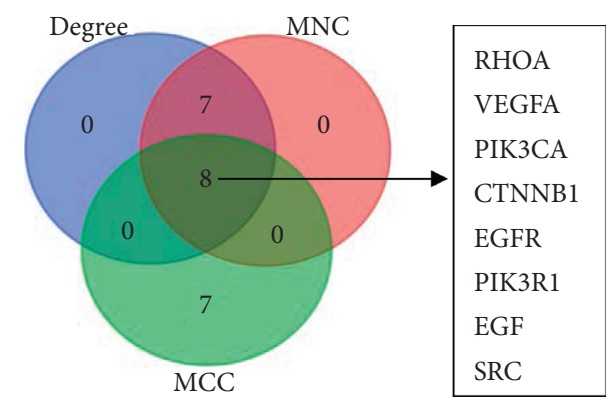

(b)

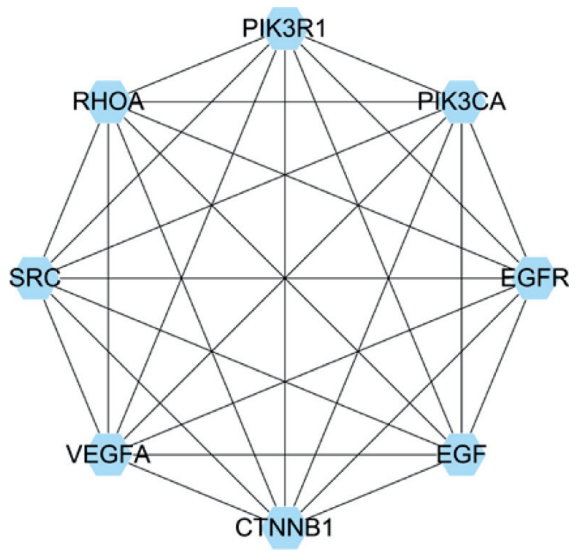

(c)

Figure 5: PPI network and hub genes. (a) PPI network of common target genes, consisting of 126 nodes and 698 edges. (b) Top 15 genes were calculated from the PPI network by the degree, MNC, and MCC, respectively. Then, the overlapping genes were screened by Venn diagrams. (c) PPI network of hub genes.

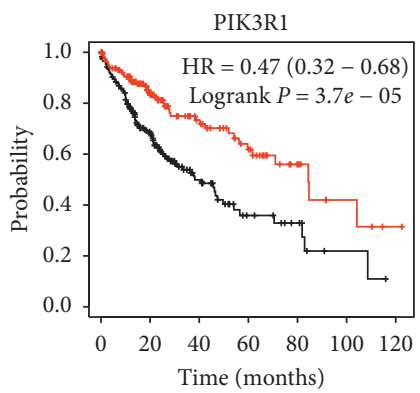

Expression

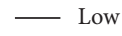

(a)
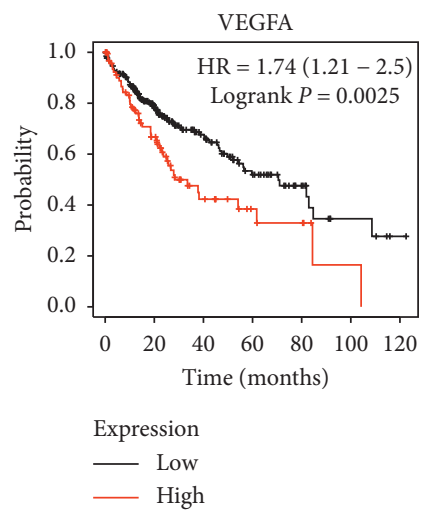
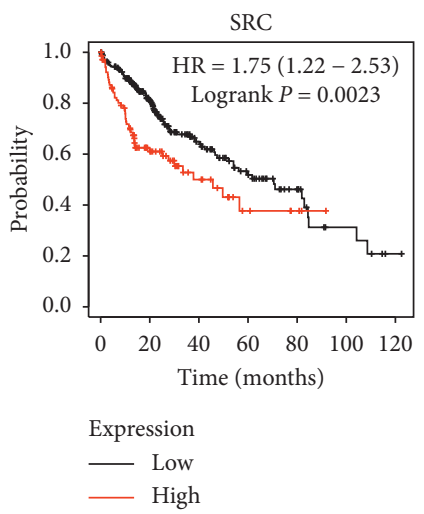

(c)

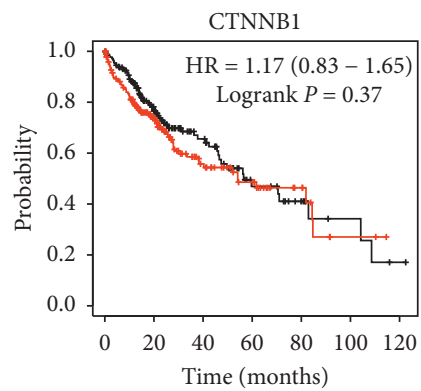

Expression

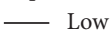

Figure 6: Continued. 


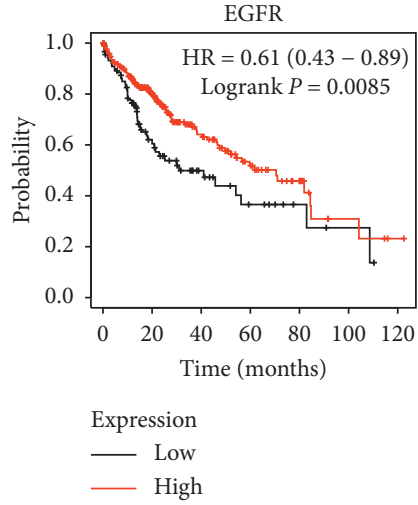

(e)

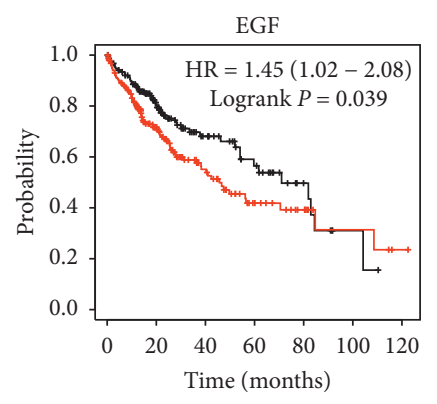

$$
\begin{gathered}
\text { Expression } \\
-\quad \text { Low } \\
\text { High }
\end{gathered}
$$

(f)
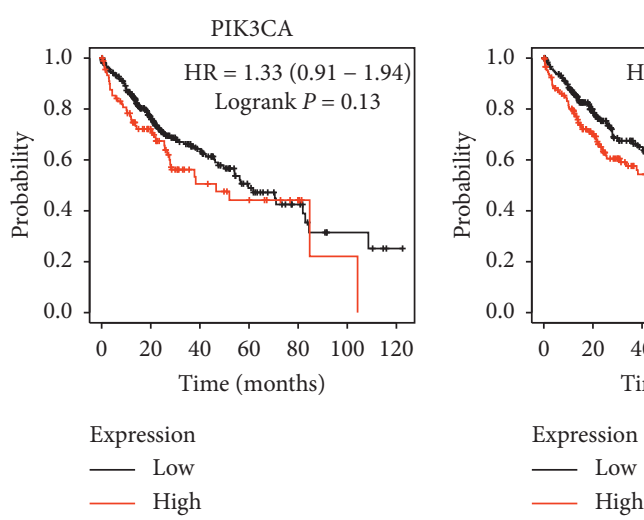

(g)

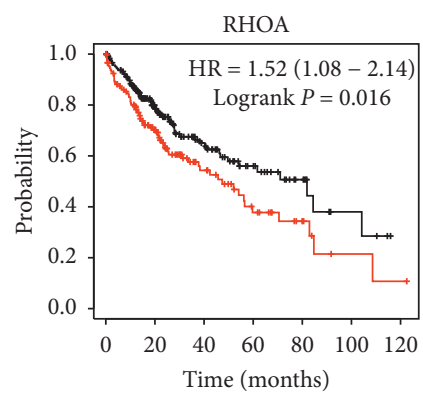

(h)

Figure 6: Prognostic values of eight hub genes for OS in patients with HCC. KM survival curves for the hub genes. High expressions of PIK3R1 (a) and EGFR (e) were associated with longer OS in HCC patients, and high expressions of VEGFA (b), SRC (c), EGF (f), and RHOA (h) were correlated with shorter OS. No significant differences were observed in other genes.

TABLE 5: Molecular docking between the target proteins and bioactive compounds of CCHs.

\begin{tabular}{lcc}
\hline Target & Compound & Affinity (kcal/mol) \\
\hline PIK3R1 & Stigmasta-5,22-dien-3-one & -9.9 \\
SRC & Stigmasta-5,22-dien-3-one & -9.4 \\
EGFR & Luteolin & -8.8 \\
EGFR & Quercetin & -8.7 \\
SRC & Pyrethrin II & -8.2 \\
RHOA & Palbinone & -8.2 \\
VEGFA & Quercetin & -7.8 \\
VEGFA & Luteolin & -7.6 \\
VEGFA & Baicalein & -7.5 \\
EGF & Luteolin & -6.0 \\
\hline
\end{tabular}

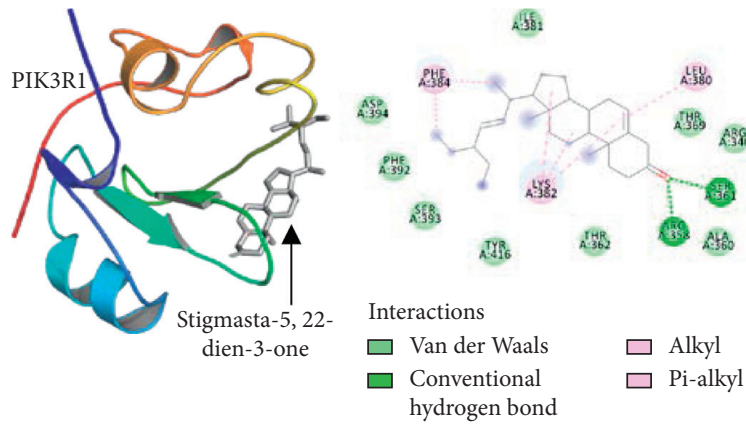

(a)

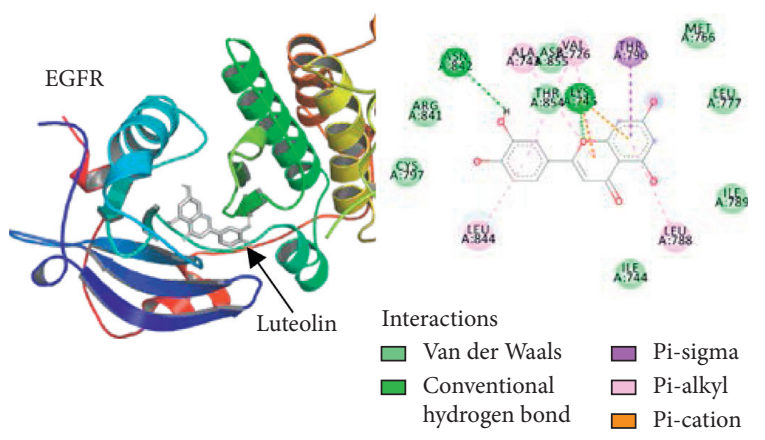

(c)

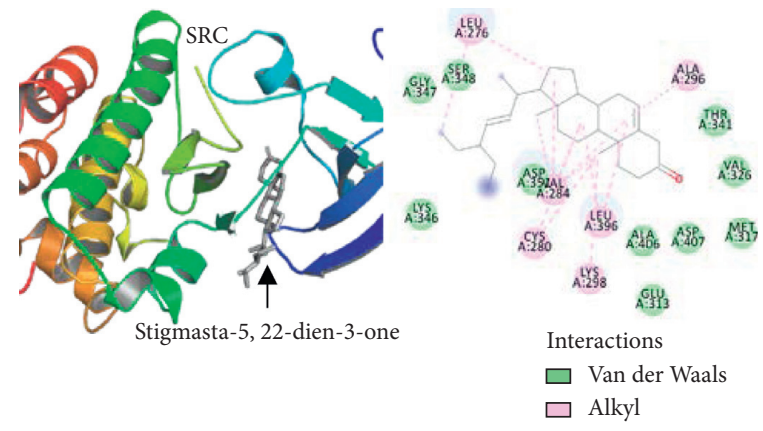

(b)
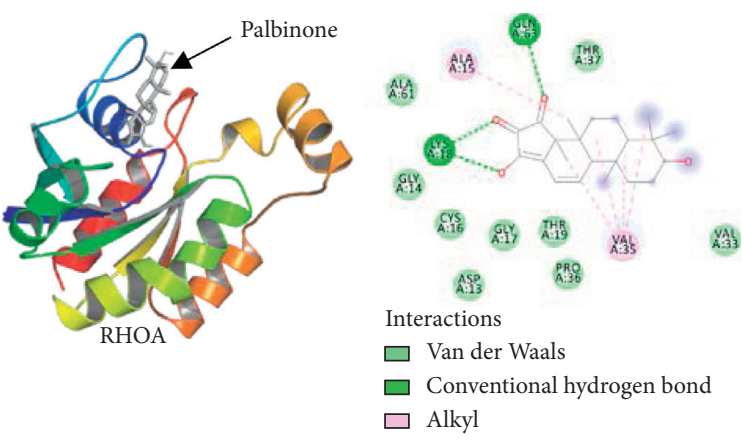

(d)

Figure 7: Continued. 


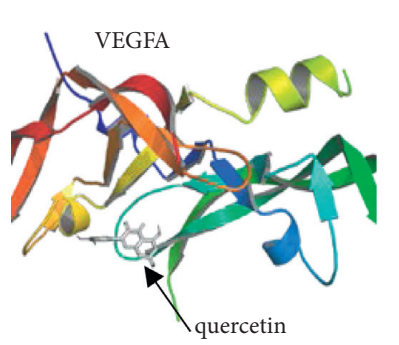

$$
\begin{aligned}
& \text { Interactions } \\
& \square \text { Van der Waals } \\
& \square \text { Conventional hydrogen bond } \\
& \square \text { Unfavorable donor-donor } \\
& \square \text { Pi-anion } \\
& \square \text { Anide-pi stacked }
\end{aligned}
$$

(e)

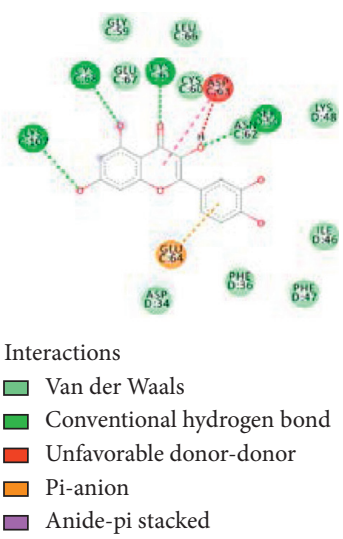

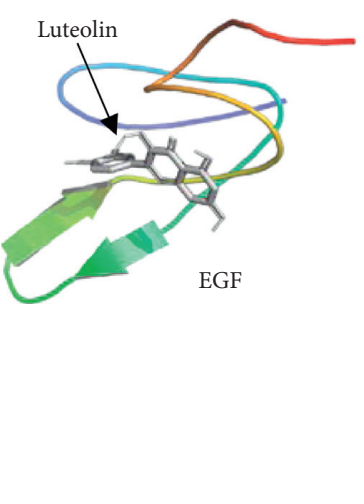

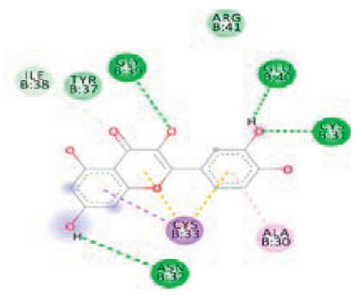

Interactions

$\square$ Van der Waals

$\square$ Conventional hydrogen bond

$\square$ Carbon hydrogen bond

$\square$ Pi-sigma

$\square$ Pi-sulfur

$\square$ Pi-alkyl

Figure 7: Molecular docking of bioactive compounds with target proteins. (a-f) The first-ranked combinations in each target protein are displayed. The 3D structures of protein receptors of key target genes and ligands (bioactive compounds) are shown in cartoon styles and gray sticks, respectively. The interactions between the protein receptors and compounds of CCHs are displayed in 2D diagrams.

instance, Figure 7(a) indicates that two hydrogen bonds between stigmasta-5,22-dien-3-one and amino acid residues (ARG-358 and SER-361) of PIK3R1 protein were generated. In addition, the van der Waals force, alkyl, and $\pi$-alkyl interactions between stigmasta-5,22-dien-3-one and other residues also play an important role. The interpretation of other molecular docking results could use this similar method. These results have successfully validated the network pharmacology data from the perspective of molecular interactions. However, this paper lacks biological experimental confirmation. Both in vivo and in vitro experiments are required to validate our results, and we should take it into consideration in future work. Last but not least, there are few reports about some bioactive compounds (e.g., stigmasta-5,22-dien-3-one, pyrethrin II, palbinone), and further research of these compounds is still needed.

\section{Conclusions}

To sum up, based on a combination of TCM prescription data mining, network pharmacology, KM survival analysis, and molecular docking, we identified eight $\mathrm{CCHs}$ for treating $\mathrm{HCC}$ and found that CCHs may play a therapeutic role in HCC by regulating the genes and pathways associated with cancer occurrence and development, angiogenesis, metastasis, and prognosis.

\section{Data Availability}

The data that support the findings of our study are openly available in http://tcmspw.com/tcmsp.php, http://www.tcmip. $\mathrm{cn} /, \quad$ http://bionet.ncpsb.org/batman-tcm/, https://www. uniprot.org/, https://www.malacards.org/, https://omim.org/, https://david.ncifcrf.gov/, https://string-db.org/, http://kmplot. com/analysis/, and https://www.rcsb.org/. The data used and analyzed during the current study are available from the corresponding author on reasonable request.

\section{Conflicts of Interest}

The authors declare that they have no conflicts of interest regarding the publication of this paper.

\section{Acknowledgments}

The authors would like to thank the TCMSP, TCMIP, BATMAN-TCM, OMIM, MalaCards, STRING, DAVID, and Kaplan-Meier plotter database for providing open access.

\section{References}

[1] F. Bray, J. Ferlay, I. Soerjomataram, R. L. Siegel, L. A. Torre, and A. Jemal, "Global cancer statistics 2018: GLOBOCAN estimates of incidence and mortality worldwide for 36 cancers in 185 countries," CA: A Cancer Journal for Clinicians, vol. 68, no. 6, pp. 394-424, 2018.

[2] F. Duan, Y.-Y. Wang, D.-G. Xu et al., "Feasibility of terahertz imaging for discrimination of human hepatocellular carcinoma," World Journal of Gastrointestinal Oncology, vol. 11, no. 2, pp. 153-160, 2019.

[3] H. B. El-Serag, "Hepatocellular carcinoma," New England Journal of Medicine, vol. 365, no. 12, pp. 1118-1127, 2011.

[4] Y. Inokawa, S. Nomoto, M. Hishida et al., "Detection of doublecortin domain-containing 2 (DCDC2), a new candidate tumor suppressor gene of hepatocellular carcinoma, by triple combination array analysis," Journal of Experimental \& Clinical Cancer Research, vol. 32, no. 1, p. 65, 2013.

[5] J. Liu, X. Li, J. Huang, and Y. Liu, "Matrix metalloproteinase 2 knockdown suppresses the proliferation of HepG2 and Huh7 cells and enhances the cisplatin effect," Open Medicine, vol. 14, no. 1, pp. 384-391, 2019.

[6] L. Zhang, C. Y. Wu, Y. Zhang et al., "Comparison of efficacy and toxicity of traditional Chinese medicine (TCM) herbal mixture LQ and conventional chemotherapy on lung cancer metastasis and survival in mouse models," Plos One, vol. 9, no. 10, 2014.

[7] S.-Y. Xi and G. Y. Minuk, "Role of traditional Chinese medicine in the management of patients with hepatocellular 
carcinoma," World Journal of Hepatology, vol. 10, no. 11, pp. 799-806, 2018.

[8] B. Hu, H.-M. An, S.-S. Wang, J.-J. Chen, and L. Xu, "Preventive and therapeutic effects of Chinese herbal compounds against hepatocellular carcinoma," Molecules, vol. 21, no. 2, p. 142, 2016.

[9] Z. Wang, J. Li, Y. Ji et al., "Traditional herbal medicine: a review of potential of inhibitory hepatocellular carcinoma in basic research and clinical trial," Evidence-Based Complementary and Alternative Medicine, vol. 2013, Article ID 268963, 7 pages, 2013.

[10] S. Liang, Y. Zou, J. Gao et al., "The Chinese medicine, jiedu recipe, inhibits the epithelial mesenchymal transition of hepatocellular carcinoma via the regulation of $\operatorname{Smad} 2 / 3$ dependent and independent pathways," Evidence-Based Complementary and Alternative Medicine, vol. 2018, Article ID 5629304, 8 pages, 2018.

[11] Z. Shi, T. Song, Y. Wan et al., "A systematic review and metaanalysis of traditional insect Chinese medicines combined chemotherapy for non-surgical hepatocellular carcinoma therapy," Scientific Reports, vol. 7, no. 1, p. 4355, 2017.

[12] S. H. Tang, D. Shen, and H. J. Yang, "Analysis on composition rules of Chinese patent drugs treating pain-related diseases based on data mining method," Chinese Journal of Integrative Medicine, vol. 25, no. 11, pp. 861-866, 2019.

[13] B. Pan, X. Shi, T. Ding, and L. Liu, "Unraveling the action mechanism of polygonum cuspidatum by a network pharmacology approach," American Journal of Translational Research, vol. 11, no. 11, pp. 6790-6811, 2019.

[14] J. L. Ru, P. Li, J. N. Wang et al., "TCMSP: a database of systems pharmacology for drug discovery from herbal medicines," Journal of Cheminformatics, vol. 6, no. 1, 2014.

[15] H.-Y. Xu, Y.-Q. Zhang, Z.-M. Liu et al., "ETCM: an encyclopaedia of traditional Chinese medicine," Nucleic Acids Research, vol. 47, no. D1, pp. D976-D982, 2019.

[16] Z. Liu, F. Guo, Y. Wang et al., "BATMAN-TCM: a Bioinformatics analysis Tool for molecular mechANism of traditional Chinese medicine," Scientific Reports, vol. 6, no. 1, p. 21146, 2016.

[17] W. J. Song, S. L. Ni, Y. L. Fu, and Y. Wang, "Uncovering the mechanism of Maxing Ganshi Decoction on asthma from a systematic perspective: a network pharmacology study," Scientific Reports, vol. 8, no. 1, p. 17362, 2018.

[18] N. Rappaport, M. Twik, I. Plaschkes et al., "MalaCards: an amalgamated human disease compendium with diverse clinical and genetic annotation and structured search," Nucleic Acids Research, vol. 45, no. D1, pp. D877-D887, 2017.

[19] J. S. Amberger, C. A. Bocchini, A. F. Scott, and A. Hamosh, "OMIM.org: leveraging knowledge across phenotype-gene relationships," Nucleic Acids Research, vol. 47, no. D1, pp. D1038-D1043, 2019.

[20] G. Dennis, B. T. Sherman, D. A. Hosack et al., "DAVID: database for annotation, visualization, and integrated discovery," Genome Biology, vol. 4, no. 5, 2003.

[21] D. Szklarczyk, A. Franceschini, S. Wyder et al., "STRING v10: protein-protein interaction networks, integrated over the tree of life," Nucleic Acids Research, vol. 43, no. D1, pp. D447-D452, 2015.

[22] C.-H. Chin, S.-H. Chen, H.-H. Wu, C.-W. Ho, M.-T. Ko, and C.-Y. Lin, "cytoHubba: identifying hub objects and sub-networks from complex interactome," BMC Systems Biology, vol. 8, no. Suppl 4, p. S11, 2014.

[23] X. Yang, Y. Li, R. Lv et al., "Study on the multitarget mechanism and key active ingredients of Herba siegesbeckiae and volatile oil against rheumatoid arthritis based on network pharmacology," Evidence-Based Complementary and Alternative Medicine, vol. 2019, Article ID 8957245, 15 pages, 2019.

[24] B. Gyorffy, P. Surowiak, J. Budczies, and A. Lanczky, "Online survival analysis software to assess the prognostic value of biomarkers using transcriptomic data in non-small-cell lung cancer," Plos One, vol. 8, no. 12, p. e82241, 2013.

[25] P. W. Rose, A. Prlić, A. Altunkaya et al., "The RCSB protein data bank: integrative view of protein, gene and 3D structural information," Nucleic Acids Research, vol. 45, no. D1, pp. D271-D281, 2017.

[26] X. Ruan, P. Du, K. Zhao et al., "Mechanism of Dayuanyin in the treatment of coronavirus disease 2019 based on network pharmacology and molecular docking," Chinese Medicine, vol. 15 , no. 1 , p. $62,2020$.

[27] O. Trott and A. J. Olson, "AutoDock Vina: improving the speed and accuracy of docking with a new scoring function, efficient optimization, and multithreading," Journal of Computational Chemistry, vol. 31, no. 2, pp. 455-461, 2010.

[28] W. Dai, Q. Gao, J. Qiu, J. Yuan, G. Wu, and G. Shen, "Quercetin induces apoptosis and enhances 5-FU therapeutic efficacy in hepatocellular carcinoma," Tumor Biology, vol. 37, no. 5, pp. 6307-6313, 2016.

[29] H. Xu, T. Yang, X. Liu et al., "Luteolin synergizes the antitumor effects of 5-fluorouracil against human hepatocellular carcinoma cells through apoptosis induction and metabolism," Life Sciences, vol. 144, pp. 138-147, 2016.

[30] X. Liu, S. Tian, M. Liu, L. Jian, and L. Zhao, "Wogonin inhibits the proliferation and invasion, and induces the apoptosis of HepG2 and Bel7402 HCC cells through NF- $\kappa$ B/Bcl-2, EGFR and EGFR downstream ERK/AKT signaling," International Journal of Molecular Medicine, vol. 38, no. 4, pp. 1250-1256, 2016.

[31] G. Zhu, X. Liu, H. Li et al., "Kaempferol inhibits proliferation, migration, and invasion of liver cancer HepG2 cells by downregulation of microRNA-21," International Journal of Immunopathology and Pharmacology, vol. 32, Article ID 2058738418814341, 2018.

[32] Y.-S. Kim, X.-F. Li, K.-H. Kang, B. Ryu, and S. K. Kim, "Stigmasterol isolated from marine microalgae Navicula incerta induces apoptosis in human hepatoma HepG2 cells," BMB Reports, vol. 47, no. 8, pp. 433-438, 2014.

[33] B. Bie, J. Sun, Y. Guo et al., "Baicalein: a review of its anticancer effects and mechanisms in Hepatocellular Carcinoma," Biomedicine \& Pharmacotherapy, vol. 93, pp. 1285-1291, 2017.

[34] Z. Zhang, Y. Xing, G. Hu, and S. Xie, "Antiproliferative effects mechanism of beta-sitosterul in hepatoma HepG2 cells," Zhongguo Zhong Yao Za Zhi Zhongguo Zhongyao Zazhi China Journal of Chinese Materia Medica, vol. 36, no. 15, pp. 2145-2148, 2011.

[35] J. Meng, X. Ai, Y. Lei et al., "USP5 promotes epithelialmesenchymal transition by stabilizing SLUG in hepatocellular carcinoma," Theranostics, vol. 9, no. 2, pp. 573-587, 2019.

[36] P. Jain, N. Kumar, V. R. Josyula et al., "A study on the role of $(+)$-catechin in suppression of HepG2 proliferation via caspase dependent pathway and enhancement of its in vitro and in vivo cytotoxic potential through liposomal formulation," European Journal of Pharmaceutical Sciences, vol. 50, no. 3-4, pp. 353-365, 2013.

[37] A. Psyrri, N. Arkadopoulos, M. Vassilakopoulou, V. Smyrniotis, and G. Dimitriadis, "Pathways and targets in hepatocellular carcinoma," Expert Review of Anticancer Therapy, vol. 12, no. 10, pp. 1347-1357, 2012. 
[38] S. Mikhail, D. Cosgrove, and A. Zeidan, "Hepatocellular carcinoma: systemic therapies and future perspectives," $E x$ pert Review of Anticancer Therapy, vol. 14, no. 10, pp. 12051218, 2014.

[39] H.-I. Yang, S.-N. Lu, Y.-F. Liaw et al., "Hepatitis B e antigen and the risk of hepatocellular carcinoma," New England Journal of Medicine, vol. 347, no. 3, pp. 168-174, 2002.

[40] R. Chauhan, N. D. Churchill, P. M. Mulrooney-Cousins, and T. I. Michalak, "Initial sites of hepadnavirus integration into host genome in human hepatocytes and in the woodchuck model of hepatitis B-associated hepatocellular carcinoma," Oncogenesis, vol. 6, no. 4, p. e317, 2017.

[41] Y. Tanaka, R. Tateishi, and K. Koike, "Proteoglycans are attractive biomarkers and therapeutic targets in hepatocellular carcinoma," International Journal of Molecular Sciences, vol. 19, no. 10, 2018.

[42] J. Lin, W. Lin, Y. Ye et al., "Kindlin-2 promotes hepatocellular carcinoma invasion and metastasis by increasing Wnt/ $\beta$-catenin signaling," Journal of Experimental and Clinical Cancer Research, vol. 36, no. 1, p. 134, 2017.

[43] C. Hayes and K. Chayama, "MicroRNAs as biomarkers for liver disease and hepatocellular carcinoma," International Journal of Molecular Sciences, vol. 17, no. 3, p. 280, 2016.

[44] F. Deng, D. Chen, X. Wei et al., "Development and validation of a prognostic classifier based on HIF-1 signaling for hepatocellular carcinoma," Aging, vol. 12, no. 4, pp. 3431-3450, 2020.

[45] P. Manka, J. D. Coombes, R. Boosman, K. Gauthier, S. Papa, and W. K. Syn, "Thyroid hormone in the regulation of hepatocellular carcinoma and its microenvironment," Cancer Letters, vol. 419, pp. 175-186, 2018.

[46] X. Zhang, H. Zhang, and L. Ye, "Effects of hepatitis B virus X protein on the development of liver cancer," Journal of Laboratory and Clinical Medicine, vol. 147, no. 2, pp. 58-66, 2006.

[47] B. Delire and P. Stärkel, "The Ras/MAPK pathway and hepatocarcinoma: pathogenesis and therapeutic implications," European Journal of Clinical Investigation, vol. 45, no. 6, pp. 609-623, 2015.

[48] W. Liu, T.-F. Guo, Z.-T. Jing et al., "Hepatitis B virus core protein promotes hepatocarcinogenesis by enhancing Src expression and activating the Src/PI3K/Akt pathway," The FASEB Journal, vol. 32, no. 6, pp. 3033-3046, 2018.

[49] Y. Bai, F. Xie, F. Miao et al., "The diagnostic and prognostic role of RhoA in hepatocellular carcinoma," Aging, vol. 11, no. 14, pp. 5158-5172, 2019.

[50] X. Ai, L. Xiang, Z. Huang et al., "Overexpression of PIK3R1 promotes hepatocellular carcinoma progression," Biological Research, vol. 51, no. 1, p. 52, 2018.

[51] Z. Liu, D. Chen, F. Ning, J. Du, and H. Wang, "EGF is highly expressed in hepatocellular carcinoma (HCC) and promotes motility of HCC cells via fibronectin," Journal of Cellular Biochemistry, vol. 119, no. 5, pp. 4170-4183, 2018.

[52] R. Panvichian, A. Tantiwetrueangdet, P. Sornmayura, and S. Leelaudomlipi, "Missense mutations in exons 18-24 of EGFR in hepatocellular carcinoma tissues," BioMed Research International, vol. 2015, Article ID 171845, 7 pages, 2015.

[53] K. Komposch and M. Sibilia, "EGFR signaling in liver diseases," International Journal of Molecular Sciences, vol. 17, no. 1, 2015.

[54] J. Lin, S. Cao, Y. Wang et al., "Long non-coding RNA UBE2CP3 enhances HCC cell secretion of VEGFA and promotes angiogenesis by activating ERK1/2/HIF-1 $\alpha /$ VEGFA signalling in hepatocellular carcinoma," Journal of
Experimental and Clinical Cancer Research, vol. 37, no. 1, p. 113, 2018.

[55] J.-J. Yan, Y.-N. Zhang, J.-Z. Liao et al., "MiR-497 suppresses angiogenesis and metastasis of hepatocellular carcinoma by inhibiting VEGFA and AEG-1," Oncotarget, vol. 6, no. 30, pp. 29527-29542, 2015. 